Micro Electro Mechanical Systems '92

Travemünde (Germany), February 4-7, 1992

\title{
POLYMER BONDING OF MICRO-MACHINED SILICON STRUCTURES
}

\author{
C. den Besten, R.E.G. van Hal, J. Muñoz and P. Bergveld \\ MESA Research Institute, University of Twente \\ P.O. Box 217 \\ 7500 AE Enschede \\ The Netherlands
}

\begin{abstract}
Polymer bonding is an indirect bonding technique, which can be performed at temperatures of less than $150^{\circ} \mathrm{C}$.

Thin layers of negative photoresist, polyimide and epoxy were used to bond micromachined test devices to other pieces of silicon. The strength of the resulting bond as well as the influence of primers like APS and HMDS on the bond strength were tested.
\end{abstract}

The best results have been achieved with negative photoresist without primer at a curing temperature of $130^{\circ} \mathrm{C}$. The bond strength was more than $130 \mathrm{~kg} / \mathrm{cm}^{2}$.

\section{INTRODUCTION}

Bonding of silicon to silicon becomes more and more important in modern sensor and actuator processing techniques. Because most present bonding techniques require relative high temperatures and some of them require in addition high voltages, problems occur with previously deposited metal layers and integrated electronics. Therefore, it would be convenient if a more mild bonding technique would be developed, which allows application in a later stage of sensor processing.

We have done research in order to find a bonding method, which can be used to bond micro-machined silicon sensor parts, even if the surfaces of the parts are relatively rough. This bonding technique should not degrade metallization patterns and electrical structures, which may be integrated in the sensor parts.

In the next section we shall describe the general demands of bonding techniques and after that we discuss the already existing bonding techniques. Then a new bonding technique is presented and the experimental work is described, as well as the achieved results. Finally the results are discussed and conclusions are drawn.

\section{GENERAL BONDING DEMANDS}

Any good bonding technique has to result in a relative strong bond achieved at the lowest possible temperature. Higher temperatures may damage metallization layers and cause considerable stress in the bonded materials.

Furthermore, if electrical structures are integrated in one or both to be bonded sensor parts, one has to be sure that the electrical behaviour of the sensor will not be influenced by the bonding process. In general it can be said that diffusion of metal ions in or into the silicon has to be avoided. Diffusion may occur due to high temperature or high electric fields.

The bonding conditions should not be critical in order to keep the bonding process as simple as possible.

\section{PRESENT BONDING TECHNIQUES}

Last decade an increasing number of papers about wafer bonding have been published. We may divide them into two groups according to the bonding technique, which is used: direct bonding and indirect bonding. The most commonly used techniques will be 
discussed with respect to the requirements as discussed in the previous section.

\section{Direct bonding}

Direct bonding of silicon to silicon (Silicon Fusion Bonding) is based on a chemical reaction between $\mathrm{OH}$-groups, which are present at the surface of the (native) $\mathrm{SiO}_{2}$. This reaction occurs already at $200^{\circ} \mathrm{C}$. At higher temperatures the number of $\mathrm{OH}$-groups involved in the reaction increases, which results in an increasing bond strength. At temperatures of more than $1000^{\circ} \mathrm{C}$ the bond strength may approach the strength of the bulk. $[1,2,3]$

The quality of the bond depends not only on the bonding temperature, but also on the roughness of the surfaces. Therefore, beforehand polishing of the surfaces is very important to achieve a strong, uniform bond.

The direct bonding technique cannot be used after integration of electronics in sensors, because the electrical properties and the metallization may be affected due to the relatively high bonding temperature.

Recently direct bonding at temperatures between 200 and $300^{\circ} \mathrm{C}$ has been performed [4]. In order to achieve a strong bond at these low temperatures, the surfaces were modified by spinning a thin sodiumsilicate layer on top of it. This surface modification results in a bond strength, which is comparable to that of conventional direct bonding at $1000^{\circ} \mathrm{C}$. However, a disadvantage of this technique is the presence of sodium-ions, which may diffuse into the silicon and affect the electrical properties of the electronic circuitry.

\section{Indirect bonding}

The difference between the indirect bonding and the direct bonding technique is the use of an intermediate layer of glass or metal, which is used to realize the bond between the two silicon surfaces. The most commonly used techniques are: anodic bonding, low-temperature glass bonding and eutectic bonding. The average bonding temperature of the indirect bonding techniques is lower than the bonding temperature of the high temperature direct bonding technique.

\section{1) Anodic bonding}

An intermediate layer of sodium-rich glass is bonded to the silicon surfaces due to an electric field, which is caused by a potential difference between the two surfaces. The bonding temperature is typically $300-500^{\circ} \mathrm{C}$, while the applied potential difference is typically $100-300$ Volts. The bonding mechanism is not completely understood, but it is suggested that the bonding is a result of the diffusion of sodium and oxygen ions through the glass to the cathode and anode respectively. At the interfaces a chemical reaction results in a bond between the glass and the silicon. $[5,6,7]$

Anodic bonding cannot easily be used in combination with micromachined sensors, because the intermediate layer has to be deposited at the bonding surfaces without affecting the micromachined structures. This can be realized by additional lithographic and etching steps, but this makes the bonding technique more complex.

Another disadvantage of this technique is that the electrical properties of the device may be affected due to the electric field during the bonding process and the diffusion of sodium into the silicon.

\section{2) Low temperature glass bonding}

This bonding technique makes use of an intermediate layer of boron-glass with a low melting temperature $[8,9]$. This layer can be sputtered or deposited by a CVD-process. The bonding mechanism is quite simple. The silicon wafers are heated until the glass layer melts and forms a bond with the silicon surfaces. The bonding temperature for boron-glass is $450^{\circ} \mathrm{C}$. Research is focused on finding new glasses with lower melting temperatures.

As with the other techniques the bonding temperature is too high and like the anodic bonding technique it is difficult to perform in combination with micromachined silicon devices.

\section{3) Eutectic bonding}

The intermediate layer of the eutectic bonding technique consists of a metal. The mechanism is based on the phenomena that the melting temperature of an alloy of a metal and silicon can be lower than the melting temperature of the individual materials. The lowest melting point of the alloy (the eutectic point) 
depends on the proportions of the materials in the alloy.

Gold is commonly used to bond silicon to silicon. The eutectic point lies at $370^{\circ} \mathrm{C}$. To obtain a good bond quality it is necessary to remove all the oxide of the silicon surface [10].

The main disadvantage of this technique, apart from the still relative high temperature, is the diffusion of metal atoms into the silicon. Especially gold influences strongly the electrical properties of electronic devices. Therefore the application of this technique is limited.

\section{POLYMER BONDING}

The research has been focused on an indirect bonding technique, where the intermediate layer consist of a polymer. The use of a polymer as the bonding substance has several advantages:

- The bonding temperature can be low. Most polymers polymerize at temperatures below $150^{\circ} \mathrm{C}$.

- Polymer layers do not contain metal ions, which may diffuse into the silicon.

- The bond strength can be very high. [11]

- The elastic properties after polymerization reduces the stress in the bonded materials caused by the bonding process.

The use of polymers has also some disadvantages:

- Indirect bonding with polymers will not lead to an hermetic seal.

- Not all polymers may be compatible with the conventional IC-processing techniques.

\section{Primers}

As will be obvious, the degree of coverage as well as the adhesion of the polymer to the $\mathrm{SiO}_{2}$ determine the bond strength. Silylation reagens are commonly used to improve these parameters. The coverage can be improved by using a primer like hexamethyldisilazane (HMDS), which creates a hydrophobic surface on the oxide. For some applications $\gamma$-aminopropylsilane (APS) is used as a primer, because it can react with $\mathrm{SiO}_{2}$ and polyimide or unpolymerized epoxy. The resulting chemical bond is in general stronger than a physical bond.

\section{Bonding substances}

Our research has been focused on the bonding properties of three different polymers: negative photoresist, polyimide and epoxy.

\section{1) Negative photoresist}

Negative photoresist is commonly used for photolithographic processes and is compatible with several IC-processing techniques. The elasticity of this polymer after polymerization reduces the internal stress of the bonded silicon pieces. HMDS is recommended by the manufacturer as a primer, because it provides a better coverage of $\mathrm{SiO}_{2}$ by the resist.

\section{2) Polyimide}

Polyimide is used for packaging of devices, because of the elastic properties of the polymerized layer, which reduces the stress in the device due to the packaging. It is known that the adhesion of polyimide to $\mathrm{SiO}_{2}$ is good, therefore we decided to test the bonding properties of this polymer. In addition APS is used as a primer, because it forms a chemical bond with polyimide as well as with the $\mathrm{SiO}_{2}$. This should result in a higher bond strength.

\section{3) Epoxy}

The use of epoxy to glue materials is well known. The bond strength of these polymers can be very high [11]. To obtain thin epoxy layers it is necessary to make a diluted solution. The solvent we used was n-methylpyrrolidon (NMP). Toluene was added to the solution to stimulate the evaporation of NMP. As in the case of polyimide APS forms a chemical bond with $\mathrm{SiO}_{2}$ and epoxy, therefore we decided to use APS as a primer.

\section{EXPERIMENTS}

To test the polymer bonding first test devices have been realised consisting of a $10 \times 10 \mathrm{~mm}^{2}$ piece of silicon in which four cavities are etched of $1 \mathrm{~mm}$ wide, $7 \mathrm{~mm}$ long and 60 micron deep using $\mathrm{SiO}_{2}$ as a mask. After the etching of the cavities all $\mathrm{SiO}_{2}$ was removed, so the bonding surface was only covered by a thin layer of native oxide.

The micromachined side of the test devices was coated with a thin layer of the bonding substance. This 
was accomplished by spinning the bonding substance on a separate bare silicon wafer, pressing the testdevice on the wafer and then gently pulling this wafer and the test device apart. This method is also used by Smith [12] and is similar to the ordinary stamp. After that the test device was pressed on a piece of bare silicon of the same dimensions as the test device and heated for a short period to complete the bonding process. If necessary both pieces of silicon were pretreated with a primer.

We tested the following samples:

sample substance (+ primer)

1. Neg. photoresist (Waycoat 3IC, Olin Hunt)

2. Neg. photoresist (Waycoat 3IC, Olin Hunt)

3. Neg. photoresist (Waycoat 3IC) + HMDS

4. Neg. photoresist (Waycoat 3IC) + HMDS

$5 . \quad$ Polyimide (PI2555, DuPont)

6. Polyimide (PI2555, DuPont)

7. Polyimide (PI2555, DuPont) + APS

8. Polyimide (PI2555, DuPont) + APS

9. Epoxy (C8W795, Dexter)

10. Epoxy (C8W795, Dexter)

11. Epoxy (C8W795, Dexter) + APS

12. Epoxy (C8W795, Dexter) + APS

13. Epoxy (C8W795, Dexter) + APS

14. Epoxy (C8W795, Dexter) + APS

The polyimide solution in samples 5 up to 8 consisted of a solution of polyimide and NMP in volume proportions 1:3. The epoxy solution in the samples 9 up to 12 was composed of $2.0 \mathrm{~g}$. of resin, $0.5 \mathrm{~g}$ of hardner, $25 \mathrm{ml}$. NMP and $15 \mathrm{ml}$. toluene. In the samples 13 and 14 the epoxy solution was composed of $2.0 \mathrm{~g}$. of resin, $0.5 \mathrm{~g}$. of hardner, $38 \mathrm{ml}$. NMP and 22 $\mathrm{ml}$. toluene.

The samples 1 up to 8 were cured for 30 minutes at $130^{\circ} \mathrm{C}$. The samples 9 up to 14 were cured for 30 minutes at $90^{\circ} \mathrm{C}$.

The thickness of the intermediate layer was measured on bare pieces of silicon which were partly coated with the bonding substance following the same stamp procedure as the test devices. A surface profile scanner (Dektak 3030) was used to determine the average thickness of the layer.

The peeltests were performed at a computer controlled set-up (MTS 810) at the faculty of Mechanical Engineering of the University of Twente. The maximal force which was applied to the bond before fracture was stored automatically.

\section{RESULTS}

Thickness measurement

\begin{tabular}{|r|c|c|c|}
\hline sample & $\begin{array}{c}\text { spin speed } \\
(\mathrm{rpm})\end{array}$ & $\begin{array}{c}\text { spin time } \\
(\mathrm{s})\end{array}$ & $\begin{array}{c}\text { thickness } \\
(\mathrm{nm})\end{array}$ \\
\hline $1-4$ & 1500 & 20 & 1100 \\
$5-8$ & 3500 & 20 & 130 \\
$9-12$ & 3500 & 20 & 180 \\
$13-14$ & 3500 & 20 & 160 \\
\hline
\end{tabular}

\section{Peel tests}

\begin{tabular}{|c|c|c|c|}
\hline sample & $\begin{array}{c}\text { curing temp. } \\
\left({ }^{\circ} \mathrm{C}\right)\end{array}$ & $\begin{array}{c}\text { curing time } \\
(\mathrm{min})\end{array}$ & $\begin{array}{c}\text { bond strength } \\
\left(\mathrm{kg} / \mathrm{cm}^{2}\right)\end{array}$ \\
\hline 1 & 130 & 30 & 132 \\
2 & 130 & 30 & 167 \\
3 & 130 & 30 & 79 \\
4 & 130 & 30 & 50 \\
5 & 130 & 30 & - \\
6 & 130 & 30 & - \\
7 & 130 & 30 & 76 \\
8 & 130 & 30 & - \\
9 & 90 & 30 & 99 \\
10 & 90 & 30 & 60 \\
11 & 90 & 30 & - \\
12 & 90 & 30 & 88 \\
13 & 90 & 30 & 96 \\
14 & 90 & 30 & 85 \\
\hline
\end{tabular}

The samples 5 and 6 (polyimide without APS) broke before testing. The results of sample 8 and 11 were not reliable due to technical problems during the test.

\section{DISCUSSION}

\section{Bonding technique}

The photoresist layers are much thicker than the layers of polyimide and epoxy. We increased the spinning speed in order to decrease the layer thickness of the negative photoresist, but then the solvent in the resist (xylene) evaporated already during the spinning and as a result of that it was impossible to coat the test devices. Thinner layers can be achieved by adding some NMP to the photoresist solution, which hinders the evaporation of xylene, but we decided to use the standard resist as supplied by 
the manufacturer, because the layer thickness would not affect the adhesion at the $\mathrm{SiO}_{2}$-polymer interface.

The coverage of the coating at the test devices was examined optically by a microscope. The coverage of the negative photoresist was good and the complete coated area contributed to the bond, probably due to the relative thick layer. No photoresist was found in the cavities of the test devices.

The coverage of the polyimide and epoxy was also good, but mostly only a part of the coated area contributed to the bond as could be seen after the fracture of the bonded parts. A thicker layer may solve this problem. No polyimide or epoxy was found in the cavities of the devices.

\section{Peel tests}

Negative photoresist

From the results in section 6 we see that the highest bond strength is found, when negative photoresist without HMDS is used as the intermediate layer. The bond strength is lower when HMDS is used. Without HMDS the negative photoresist is bonded to the $\mathrm{OH}$-groups of the $\mathrm{SiO}_{2}$ by hydrogen bonds, which are relatively strong. HMDS is also reacting with the $\mathrm{OH}$-groups of the $\mathrm{SiO}_{2}$, so if HMDS is used as a primer negative photoresist cannot bond to the $\mathrm{OH}-g r o u p s$ anymore. In that case the adhesion is purely physical, which results in a lower bond strength.

Polyimide

Polyimide is not suited for polymer bonding unless APS is used as a primer. The physical adhesion of polyimide to the $\mathrm{SiO}_{2}$ is poor. Even small forces which were applied to the test samples during the preparation for the tests were sufficient to fracture the bond.

When the primer APS was used a bond strength of 76 $\mathrm{kg} / \mathrm{cm}^{2}$ was measured. This increased bond strength is a result of the chemical bonds between polyimide and $\mathrm{SiO}_{2}$ caused by the APS.

\section{Epoxy}

As seen in the results there is no significant difference in the bond strength of epoxy with or without APS as primer. Examining of the fractured surfaces learned that the bond was fractured in the bulk of the epoxy in stead of at the interface with the oxide as in the case of photoresist and polyimide. This implies that the physical as well as the chemical bond between epoxy and $\mathrm{SiO} 2$ is stronger then the bulk of the epoxy.

\section{CONCLUSION}

Polymer bonding provides sensor designers with a low temperature bonding technique. The best results have obviously been achieved with negative photoresist without primer at a bonding temperature of $130^{\circ} \mathrm{C}$.

\section{ACKNOWLEDGEMENT}

We wish to thank Erwin Petter for his research on this subject during his study and also Pieter van Riessen for performing the peel tests at the faculty of Mechanical Engineering of the University of Twente.

\section{REFERENCES}

[1] J.B. Lasky, "Wafer bonding for silicon-on-insulator technologies", Appl. Phys. Lett., Vol. 48, pp.78-80, 1986

[2] M. Shimbo, K. Furukawa, K. Fukuda and K. Tanzawa, "Silicon-to-silicon direct bonding method", J. Appl. Phys., Vol. 60 pp.2987-2989, 1986

[3] C. Harendt, H. Graf, E. Penteker and B. Höfflinger, "Wafer bonding: Investigation and in situ observation of the bond process", Sensors and Actuaters, Vol. A21-A23 pp.927-930, 1990

[4] H.J. Quenzer and W. Benecke, "Low-Temperature silicon wafer bonding", Eurosensors V, book of abstracts p.202, 1991

[5] G. Wallis and D.I. Pomerantz, "Field assisted glass-metal sealing", J. Appl. Phys., Vol. 40 pp.3946-3949, 1969

[6] W.Y. Lee, F. Sequenda and J. Salem, "Field-assisted bonding below $200^{\circ} \mathrm{C}$ using metal and glass thin-film interlayers", Appl. Phys. Lett., Vol. 50, pp. 522-524, 1989

[7] Y. Kanda, K. Matsuda, C. Murayama and J. Sugaya, "The mechanism fo field-assisted silicon-glass bonding", Sensors and Actuators, Vol. A21-A23 pp. 939-943, 1990

[8] L.A. Field and R.S. Muller, "Fusing silicon wafers with low melting temperature glass", Sensors and Actuators, Vol. A21-A23 pp. 935-938, 1990

[9] R. Legtenberg, S. Bouwstra and M. Elwenspoek, "Low-temperature glass bonding for sensor applications", proceedings MME-workshop, Berlin 1990 
[10] D. Hamer and J.V. Biggers, "Thick film hybrid microcircuit technology", (Wiley) pp. 195-200, 1972

[11] J. Hennaut, J. Othmezouri and J. Charlier, "Influence of heat treatments on the cohesion and adhesion strength of plasma-sprayed deposits", Thin Solid Films, Vol. 192 pp. 97-101

[12] R.L. Smith and S.D. Collins, "Micromachined packaging for chemical microsensors", IEEE Trans. on Elec. Devices, Vol. 35 pp. 787-792, 1988 